\title{
INCLUSÃO DE PESSOAS COM DEFICIÊNCIA NOS PROJETOS DE EXTENSÃO DE PRATICAS CORPORAIS DA UNIVERSIDADE FEDERAL DE GOIÁS
}

\author{
Vanessa Helena Santana Dalla Déa \\ Universidade Federal de Goiás, Goiânia, Goiás, Brasil \\ Maycon Vasconcelos Cunha \\ Universidade Federal de Goiás, Goiânia, Goiás, Brasil \\ Oromar Augusto dos Santos Nascimento \\ Universidade Federal de Goiás, Goiânia, Goiás, Brasil \\ Amanda Fonseca de Lima \\ Universidade Federal de Goiás, Goiânia, Goiás, Brasil \\ Marlini Dorneles de Lima \\ Universidade Federal de Goiás, Goiânia, Goiás, Brasil
}

\section{Resumo}

A inclusão das pessoas com deficiência nos mais diferentes contextos sociais é um direito. O presente artigo tem como objetivo apresentar como acontece a inclusão destas pessoas nos projetos de extensão da UFG, categorizando formas de inclusão e o perfil das pessoas incluídas. Como metodologia foi utilizada a observação sistemática e pesquisa de campo quantitativo-descritiva. Como resultado tem-se 25 pessoas com deficiência incluídas, sendo $80 \%$ nas praticas aquáticas, $16 \%$ nas praticas aquáticas e dança, e $4 \%$ fazem somente dança. Foram encontradas três formas diferentes de inclusão: inclusão automática e independente com $48 \%$ dos participantes, inclusão com dependência momentânea com $40 \%$ e inclusão com dependência permanente com apenas $12 \%$ dos participantes.

Palavras-chave: Método. Educação Física e Treinamento. Socialização.

\section{Introdução}

\begin{abstract}
A sociedade vem optando pelo sentido de sua existência por meio da produção, da produtividade, da máquina, e do rendimento, da maximização, da otimização, e da eficiência. A busca enlouquecida pela eficiência proporciona no homem atual a incorporação da aversão pela deficiência. Segundo Lima e Palma (2006, p.132)
\end{abstract}

desde os mais remotos tempos da civilização, a relação das pessoas que possuíam algum tipo de deficiência com as que eram aparentemente "normais" foi permeada por muitos preconceitos, estigmas e rotulações. 
Para Laplantine (1988) os seres humanos têm em comum a capacidade para se diferenciar uns dos outro. Somos todos diferentes, no entanto a sociedade ainda não respeita e aceita as diferenças. Entretanto a discussão de inclusão no campo educacional vem como um enfrentamento há uma pratica educacional hegemônica que, de certa forma, se constitui como um espelho da sociedade atual a qual incorporou o sistema neoliberal de produtividade, competividade, consumo e controle social.

A luta pelos direitos de inclusão da pessoa com deficiência vem sendo definida por meio de Leis e Diretrizes como a Declaração Universal dos Direitos Humanos (1948); a Declaração dos Direitos do Homem e do Cidadão (1789); a Declaração de Salamanca (UNESCO, 1994); entre outras tantas. No entanto além do direito adquirido a formação do professor é fundamental para que o processo de inclusão aconteça com sucesso. Aguiar e Duarte (2005) em estudo com professores de Educação Física concluíram que 97\% destes não possuíam conhecimento para incluir as pessoas com deficiências nas suas aulas. Neves (2006) apresenta em seus estudos a falta de capacitação dos professores de Educação Física da rede municipal de ensino de Goiânia.

Diante dessas considerações, destaca-se a urgência e importância de fomentar a discussão e a qualificação dos futuros professores para o trato com práticas pedagógicas na perspectiva da inclusão. Bem como fomentar o debate com a comunidade almejando uma educação inclusiva que permita ultrapassar a valorização do "normal" em termos de padronização de corpo, comportamento e aprendizagem para o respeito à diversidade possibilitando que a corporalidade seja fruto de uma interação recíproca e complexa entre os diferentes corpos que constituem a sociedade.

Em 2009 nos projetos de extensão de praticas corporais da FEF/UFG participavam mais de 800 pessoas no entanto nenhuma pessoa com deficiência estava incluída nestes projetos. Neste sentido foi criado na Faculdade de Educação Física (FEF) o "Projeto Dando Asas" que visa possibilitar a inclusão de pessoas com deficiência nos projetos de extensão de praticas corporais da Universidade Federal de Goiás (UFG), apresentando para acadêmicos dos cursos de Educação Física e Dança a oportunidade de vivenciar o paradigma da inclusão ainda durante a formação inicial. Este projeto visa: facilitar a acessibilidade, realizando apoio pedagógico e acadêmico à monitores e co- 
ordenadores dos projetos; criando adaptações quando necessárias buscando a inclusão efetiva e benéfica das pessoas com deficiência nos projetos de extensão de praticas corporais. Este trabalho tem como princípio norteador a não criar projetos exclusivos para pessoas com deficiência, que na nossa compreensão fugiriam do paradigma inclusivo, e sim proporcionar a estas o direito de inclusão nos projetos já existentes como qualquer outra pessoa criando arranjos pedagógicos para que isso aconteça. Para que se atinja este objetivo é preciso adaptar a atividade para o participante, para Rodrigues (2006, p. 54) "adaptar é adequar a exigência da tarefa ao nível de desempenho do executante".

Mais do que apenas a adaptação das atividades, para que haja a inclusão nas praticas corporais, faz se necessárias diferentes dinâmicas e estratégias de ensino, adaptação ou complementação curricular, resultando em "novo arranjo pedagógico" (LIMA; PALMA, 2006). Para que haja a inclusão nos projetos de extensão da FEF/UFG, os arranjos pedagógicos com paradigma inclusivo foram criados pelo projeto "Dando Asas" caracterizando-se em formas distintas de inclusão baseadas nas eficiências e deficiências das pessoas com deficiência incluídas nos projetos.

Esse artigo tem como objetivos investigar e apresentar as categorias e formas de efetivar a inclusão das pessoas com deficiência nos projetos de extensão de praticas aquáticas, isto é as condições de dependência ou independência durante o processo pedagógico, bem como, o perfil dos alunos com deficiência participantes dos projetos de extensão da FEF/UFG, compreendendo e considerando as especificidades de cada pratica corporal investigada.

\section{Metodologia}

Para identificar o perfil dos participantes com deficiência foram utilizados os seguintes métodos de pesquisa: pesquisa de campo quantitativo-descritiva por meio de entrevista com questionário semiestruturado (LAKATOS; MARCONI, 2001). Para entrevista foram questionados os dados de idade, gênero, tipo de deficiência, modalidade a qual escolheu participar e objetivo da participação.

As categorias de formas de inclusão foram determinadas por meio de observação sistemática, onde, segundo Lakatos e Marconi (2001, p. 193), o pesquisador busca a obtenção de determinados e específicos 
aspectos da realidade, que neste estudo foram realizadas no primeiro semestre de 2012. Após a observação as formas de inclusão foram categorizadas de acordo com similaridades do no processo utilizado para que a pessoa com deficiência pudesse participar da natação ou hidroginástica com o máximo possível de inclusão social e na pratica por ele escolhida. Outro dado apresentado neste trabalho foi a quantificação dos alunos com deficiência em cada forma de inclusão.

Os dados do perfil dos participantes e da divisão dos participantes nas formas de inclusão foram quantificados por meio de percentuais de dados comuns por meio do Excel 2007.

\section{Resultados e discussão}

Atualmente 960 pessoas fazem parte dos diferentes projetos de extensão de praticas corporais da $\mathrm{FEF} / \mathrm{UFG}$, destes 25 pessoas possuem deficiência e foram incluídas pelo projeto "Dando Asas". Nesta instituição são oferecidos projetos de extensão para comunidade externa e interna da universidade de praticas aquáticas (natação adulto, natação infantil e hidroginástica), danças (flamenco, dança do ventre, dança de rua e danças variadas), lutas (capoeira, judô e luta olímpica) e praticas corporais variadas (musculação, ginástica e ioga). As pessoas com deficiência são encaminhadas para o projeto Dando Asas e escolhem a modalidade da qual gostariam de participar.

Das pessoas com deficiência incluídas ou em processo de inclusão por meio do projeto Dando Asas, $80 \%$ fazem somente práticas aquáticas (sendo $45 \%$ pessoas do gênero masculino e $55 \%$ pessoas do gênero feminino), $4 \%$ fazem somente dança (sendo $100 \%$ do gênero feminino), e $16 \%$ fazem práticas aquáticas e dança (sendo $25 \%$ do gênero masculino $75 \%$ do gênero feminino). Das 25 pessoas com deficiência incluídas nos projetos nenhuma faz outra atividade que não seja a pratica aquática ou a dança inclusiva. Este fato nos faz refletir da necessidade de melhor atenção e incentivo para a inclusão nas outras atividades considerando que estas atividades têm características que poderiam propiciar diversos benefícios para as pessoas com deficiência.

A pratica corporal aquática (natação e hidroginástica) é a atividade mais procurada pelas pessoas com deficiência na $\mathrm{FEF} / \mathrm{UFG}$, isso se dá principalmente por características próprias do meio onde acontece esta pratica que facilita a movimentação de seus participantes, ou seja, o 
empuxo e a pressão hidrostática (DALLA DÉA; DUARTE, 2009). A predominância das práticas aquáticas em relação à dança e demais atividades é justificada pelo fato de são atividades terrestres, segundo Velasco (1997, p. 44)

a adaptação aquática, atenua a função da gravidade, razão pela qual algumas pessoas com deficiência motora atingem na água uma profunda sensação de liberdade e expandem a sua autoestima e autossegurança; quando em terra, a gravidade os aprisiona e impede de se locomoverem com autonomia.

A média de idade dos alunos do projeto é de 29,4 anos, sendo que a média de idade dos alunos que fazem somente práticas aquáticas é de 28,95 anos, a média de idade dos alunos que fazem somente dança é de 57 anos, e a média de idade dos alunos que fazem práticas aquáticas e dança é de 24,75 anos. Estes dados nos mostram que a faixa etária dos alunos incluídos pelo projeto são variadas, devido ao fato de que são oferecidas práticas corporais que despertam o interesse de todas as faixas etárias (NAKAMURA; SILVEIRA, 1998).

Em relação ao tipo de deficiência predominante, $76 \%$ dos alunos apresentaram a deficiência motora como dominante, e $24 \%$ apresentaram a deficiência intelectual como predominante, e não há nenhuma pessoa em que a deficiência sensorial seja predominante. Algumas pessoas apresentam pequenas alterações visuais e/ou auditivas, aliadas a deficiência intelectual ou motora, mas não significativas. Este fato pode demonstrar uma falha na divulgação do projeto uma vez que existe maior contato com instituições ligadas a deficiência motora e intelectual.

Em relação aos objetivos citados pelas pessoas com deficiência ao praticar a atividade: $35,71 \%$ relacionaram o objetivo com a melhora das habilidades e da coordenação relacionados à deficiência existente, $17,86 \%$ relacionaram com a manutenção ou perda de peso, $14,29 \%$ relacionaram de forma generalizada com melhoras na saúde, 10,71\% relacionaram com a melhora na sociabilização, 7,14\% relacionaram com melhora no comportamento, 3,50\% relacionaram com o aumento da autonomia, 3,57\% relacionaram com a diminuição da ansiedade, e $3,57 \%$ relacionaram com o lazer. Em estudos com pessoas sem deficiência os fatores qualidade de vida e fatores estéticos são os principais objetivos. Santos e Knijnik (2006) ao estudar adultos sem 
deficiência detectaram que a maioria (44\%) dos estudados tinham como objetivo da pratica a melhora da qualidade de vida e lazer. Tahara, Schwartz e Silva (2003) apresentam como principal objetivo na atividade física, em seus estudos, fatores estéticos e qualidade de vida.

Como já foi dito anteriormente a pratica aquática inclui a grande maioria das pessoas com deficiência incluídas nos projetos, abrangendo um total de $96 \%$, sendo que $80 \%$ fazem apenas praticas aquáticas e $16 \%$ fazem praticas aquáticas e dança. Assim neste estudo optou-se em avaliar mais sistematicamente como acontece a inclusão das pessoas com deficiência nas praticas aquáticas (natação e hidroginástica), considerando que o objetivo final é que estas pessoas participem de forma mais autônoma e incluídos no grupo.

Por meio da observação sistemática foi possível observar que três formas distintas de inclusão acontecem. Após conhecer as características do aluno com deficiência, determinando eficiências e limitações, descobrir suas preferências e objetivos, foi realizado um estudo de caso com análise criteriosa para determinar como foram os arranjos pedagógicos para que o aluno possa participar de maneira coerente, benéfica e no paradigma inclusivo. As três formas de inclusão presentes encontradas neste projeto são descritas a seguir:

1-Inclusão automática e independente: Essa inclusão acontece quando processo de adaptação da pessoa com deficiência ocorre sem a interferência de nenhum dos monitores. Ao ter seus primeiros contatos com á pratica aquática a pessoa com deficiência consegue sua autonomia, sentindo confiança quanto ao ambiente e ás atividades propostas pelo professor. Estando segura quanto a esses dois aspectos e não necessitando do acompanhamento a pessoa com deficiência esta automaticamente inclusa naquele meio. Os comprometimentos resultantes da deficiência destas pessoas não é suficiente para necessitar de grandes adaptações. Apenas algumas informações são transmitidas pelos responsáveis pelo projeto no sentido de favorecer a participação e os benefícios com segurança. Se encaixando nas especificações acima estão 12 pessoas com deficiência, que representam $48 \%$ do total de pessoas integrantes do projeto.

2-Inclusão com dependência momentânea: Esta acontece quando a pessoa com deficiência em seu processo de adaptação é acompanhada por um monitor do projeto "Dando Asas" temporariamente. Neste processo a pessoa com deficiência ao ter seu primeiro contato com o ambiente aquático não se sente confiante ou não tem autonomia na pratica recebendo acompanhamento por monitores, que a partir das 
necessidades de adaptação cria meios que auxiliam a inclusão da pessoa com deficiência. Ao conseguir sua autonomia a pessoa com deficiência deixara de ser acompanhada pelo monitor estando completamente inclusa. Se encaixando nas especificações acima estão 10 pessoas com deficiência, que representam $40 \%$ do total de pessoas integrantes do projeto.

3-Inclusão com dependência permanente: Esta inclusão acontece quando a pessoa com deficiência não consegue total autonomia nas praticas precisando ser acompanhada por um monitor permanentemente, devido á característica da deficiência ou algum outro fator, algumas vezes comportamental. Ao não conseguir uma total adaptação ao ambiente, a pessoa com deficiência recebe auxilio do monitor que de acordo com a necessidade adapta de varias formas o ambiente e/ou as atividades propostas pelo professor. As atividades respeitam a modalidade escolhida, mas caminham na direção das eficiências e deficiências motoras e intelectuais presentes. Se encaixando nas especificações acima estão 03 pessoas com deficiência, que representam $12 \%$ do total de pessoas integrantes do projeto.

Estes dados mostram que apenas uma pequena porcentagem das pessoas com deficiência incluídas nas praticas aquáticas dependem de auxílio permanente, e que a grande maioria (88\%) tem condições de atingir a independência e autonomia total durante a pratica da hidroginástica e natação.

Gorgatti et al (2004) relata que 50\% dos professores de Educação Física não gostam ou não gostariam de trabalhar com crianças com necessidades especiais, dados confirmados por Gorgatti e Rose Junior (2009). Muito deste sentimento pode ser atribuído a crença de que a pessoa com deficiência requer muito trabalho e adaptações. No entanto os resultados deste trabalho mostram que apenas $12 \%$ dos incluídos necessitaram de maiores cuidados.

\section{Considerações finais}

O projeto Dando Asas tem atingido seus objetivos uma vez que sem a criação de projetos específicos para pessoas com deficiência vem obtendo sucesso na inclusão destas em projetos de extensão já existentes. Com base nos resultados encontrados neste estudo novos encaminhamentos serão tomados no projeto, como, por exemplo, incentivar a inclusão nos demais projetos de praticas corporais e buscar a inclusão de pessoas com deficiência sensorial. 
Entendemos que a inclusão de pessoas com deficiência nos projetos e na sociedade proporciona benefícios para todos: monitores e professores envolvidos lucram com ganho de vivencias e conhecimentos, e a sociedade ganha no sentido que aprende a conviver e respeitar as diferenças. Lima e Palma (2006, p. 2) relatam que "A inclusão sugere a imagem da composição do todo e o enriquecimento pela diversidade". Cidade e Freitas (1997) relatam que a inclusão proporciona na sociedade a aceitação e valorização das diferenças, reconhecendo a diversidade humana por meio da compreensão e cooperação.

O processo de inclusão em projetos de extensão na universidade torna-se uma oportunidade de formar professores de Educação Física e Dança diferenciados com as qualidades que Ferreira (2006) destaca como necessárias em professores. Ou seja, a capacidade de compreender e praticar o acolhimento à diversidade, estando aberto a práticas inovadoras durante suas aulas.

Acreditamos que este estudo é uma rica oportunidade de analisar e repensar novas práticas pedagógicas que caminhem na contramão da exclusão.

INCLUSION OF PEOPLE WITH DISABILITIES IN THE EXTENSION PROJECTS OF BODILY PRACTICES, FEDERAL UNIVERSITY OF GOIÁS

\begin{abstract}
The inclusion of disabled people in many different social contexts is a right. This article aims to show how the inclusion of these people happens in extension projects UFG, categorizing forms of inclusion and the profile of the persons included. The methodology used was the systematic observation and fieldwork quantitativedescriptive. As a result it has 25 people with disabilities were included, and $80 \%$ in aquatic practices, $16 \%$ in water and dance practices, and only $4 \%$ are dancing. We found three different ways including: automatic inclusion and independent with $48 \%$ of participants, with inclusion momentary dependence with $40 \%$ permanent dependency and inclusion with only $12 \%$ of participants.
\end{abstract}

Keywords: Method. Physical Education and Training. Socialization.

\title{
LA INCLUSIÓN DE PERSONAS CON DISCAPACIDAD EN LOS PROYECTOS DE EXTENSIÓN DE LAS PRÁCTICAS CORPORALES, DE LA UNIVERSIDAD FEDERAL DE GOIÁS
}

\section{Resumen}

La inclusión de las personas con discapacidad en muchos contextos sociales diferentes, es un derecho. Este artículo tiene como objetivo mostrar cómo la inclusión 
de estas personas ocurre en extensión UFG proyectos, formas de categorizar la inclusión y el perfil de las personas incluidas. La metodología utilizada fue la observación sistemática y el trabajo de campo cuantitativo-descriptivo. Como resultado de ello, cuenta con 25 personas con discapacidad fueron incluidos, y el $80 \%$ en las prácticas acuáticas, el 16\% en las prácticas de agua y la danza, y sólo el $4 \%$ se baila. Encontramos tres formas diferentes, incluyendo: la inclusión automática e independiente con el $48 \%$ de los participantes, con inclusión dependencia momentánea con un $40 \%$ de dependencia permanente y la inclusión de sólo el $12 \%$ de los participantes.

Palabras clave: Método. Educación Física y Entrenamiento. Socialización.

\section{Referências}

AGUIAR, J. S.; DUARTE, E. Educação Inclusiva: um estudo na área de educação física. Revista Brasileira de Educação Especial, Marília, v.11, n.2, p. 223-240, maio/ago., 2005.

CIDADE, R. E. ; FREITAS, P. S. Noções sobre Educação Física e Esporte para pessoas portadoras de deficiência. Uberlândia: EDUFU, 1997.

DALLA DÉA, V. H. S.; DUARTE, E. Síndrome de Down: Informações, caminhos e histórias de amor. São Paulo: Phorte, 2009.

DECLARAÇÃO UNIVERSAL DOS DIREITOS HUMANOS. 1948. In: $\quad<$ http://portal.mj.gov.br/sedh/ct/legis_intern/ddh_bib_inter_universal.htm>. Acesso em: 10 de setembro de 2012 .

DECLARAÇÃO DOS DIREITOS DO HOMEM E DO CIDADÃO DE 1789. Disponível em: <http://pfdc.pgr.mpf.gov.br/atuacao-e-conteudos-de-apoio/legislacao/direitos-humanos/declar_dir_homem_cidadao.pdf>. Acesso em: 10 de setembro de 2012.

FERREIRA, W. B. Inclusão x exclusão no Brasil: reflexões sobre a formação docente dez anos após Salamanca. In: RODRIGUES, D. (Org.) Inclusão e Educação: doze olhares sobre a educação inclusiva. São Paulo: Summus, 2006. p. 212-238.

GORGATTI, M.G. et al. Atitudes dos professores de educação física do ensino regular com relação a alunos portadores de deficiência. Revista Brasileira de Ciência e Movimento, Brasília, v. 12, n. 2, p. 6368, jun., 2004. 
GORGATTI, M. G.; ROSE JÚNIOR, D. Percepções dos Professores Quanto à Inclusão de Alunos com Deficiência em Aulas de Educação Física. Movimento, Porto Alegre, v. 15, n. 2, p. 119-140, abr./jun., 2009.

LAKATOS, E.M.; MARCONI, M.A.; Fundamentos de metodologia cientifica. São Paulo: Atlas, 2001.

LAPLANTINE, F. Aprender Antropologia. Brasília: Brasiliense, 1988.

LIMA, M.D.; PALMA, L.E. Reflexões acerca dos (des) caminhos da educação física na perspectiva do paradigma da educação inclusiva in REZER, R. (Org.) O Fenômeno esportivo: ensaios críticos-reflexivos. Chapecó: Argos, 2006. p. 131-142.

NAKAMURA, O. F.; SILVEIRA, R. H. Natação para bebês. São Paulo: Ícone, 1998.

NEVES, C. P. A inclusão de pessoas com deficiência segundo professores de Educação Física na Secretaria Municipal de Educação de Goiânia. Dissertação (Mestrado em Educação)-Departamento de Educação, Universidade Católica de Goiás, Goiânia, 2006

RODRIGUES, D. As dimensões de adaptação de actividades motoras. Revista brasileira Educação Física Especial, São Paulo, v. 20, p. 5358, set., 2006. Suplemento 5.

SANTOS, S. C.; KNIJNIK, J. D. Motivos de adesão à prática de atividade física na vida Adulta intermediária1. Revista Mackenzie de Educação Física e Esporte, São Paulo, v. 5, n. 1, p. 23-34, 2006.

TAHARA, A. K.; SCHWARTZ, G. M.; SILVA, K. A. Aderência e manutenção da prática de exercícios em academias. Revista brasileira Ciência e Movimento, Brasília, v. 11, n. 4, p. 7-12, out./dez., 2003

ORGANIZAÇÃO DAS NAÇÕES UNIDAS PARA A EDUCAÇÃO, A CIÊNCIA E A CULTURA (UNESCO). Declaração de Salamanca e linha de ação. Brasília: CORDE, 1994.

VELASCO, C. G. Natação segundo a psicomotricidade. Rio de Janeiro: Sprint, 1997. 
Recebido em: 08/08/2013

Revisado em: 15/10/2013

Aprovado em: 10/11/2013

Endereço para correspondência

vanessasantana@ig.com.br

Vanessa Helena Santana Dalla Déa

Faculdade de Educação Física

Universidade Federal de Goiás

Campus Samambaia,

Caixa Postal 131 -CEP: 74001-970 Goiânia - Goiás 